\title{
Discrepancy Between Quarterly Recall and Annual Recall of Falls: A Survey of Older Adults
}

\author{
Jinho Yoo ${ }^{1}$, Sunyoung Kim², Woo-Chul Park ${ }^{1}$, Byung-Sung Kim ${ }^{1}$, Hyunrim Choi $^{1}$, Chang Won Won ${ }^{1}$ \\ ${ }^{1}$ Department of Family Medicine, College of Medicine, Kyung Hee University, Seoul, ${ }^{2}$ Department of Medicine, Graduate School, Kyung \\ Hee University, Seoul, Korea
}

Corresponding Author: Chang Won Won, MD, PhD Department of Family Medicine, Kyung Hee University Medical Center, 23 Kyunghee dae-ro, Dongdaemun-gu, Seoul 02447, Korea

Tel: $+82-2-958-8691$

Fax: +82-2-958-8699

E-mail: chunwon62@naver.com

Received: September 1, 2017 Revised: September 12, 2017 Accepted: September 26, 2017
Background: Accurate measurement of the frequency of falls is challenging because falls can only be self-reported. We hypothesized that quarterly surveys over a year would be superior to a 1-time annual survey for older adults to recall the number of falls they experienced more accurately. Methods: We recruited 317 community-dwelling older adults aged 65 years and older at a senior welfare center in Seoul, Korea. Older adults without cognitive deficit were included for follow-up. All eligible participants were surveyed via telephone every 3 months over 1-year period by trained investigators and asked to recall their total number of falls over the last 1 year at the end of the study. Results: Two hundred forty-seven participants completed all follow-ups, and 58 of them reported at least 1 fall per year. Twenty-nine participants recalled the same number of falls in 4 quarterly surveys and 1-year survey and the other 29 participants recalled differently. Participants who fell more than once $(16,55.2 \%)$ had a higher recall discrepancy than those who fell only once $(11,37.9 \%)$ according to the sum of quarterly surveys. Among 58 fallers, 56 reported falling in quarterly surveys, and 47 reported falling in the 1-year survey, indicating an approximately 19\% reduction in the rate of recall in the 1-year survey. Conclusion: Repeated surveys with a shorter recall period of 3 months or less may yield a more accurate measurement of falls than a survey with a recall period of 1 year.

Key Words: Accidental falls, Mental recall, Frail elderly, Surveys and questionnaires

\section{INTRODUCTION}

Falls are the most common cause of fatal and nonfatal injuries, and the leading cause of deaths from injury in older adults $^{1)}$. Up to one-third of older adults fall at least once per year ${ }^{2,3}$, and $20 \%-30 \%$ of them experience mild to severe injuries, constituting 10\%-15\% of all emergency department visits ${ }^{3,4)}$. Although most resulting injuries are minor, about $3 \%-5 \%$ of fallers require hospitalization ${ }^{1,5)}$ and experience heavy economic burden, especially in the case of fractures ${ }^{4,6)}$. Hip fractures are the most traumatic consequence, with a 1 -year mortality as high as $36 \%{ }^{7)}$, and only $41 \%$ to $67 \%$ of patients regain their prefracture ambulatory ability within 1 year $^{8-10)}$. Minor injuries can also affect the quality of life by inducing a fear of falling, which can lead to restriction of activities, social isolation, and depression ${ }^{11-13)}$.

Despite the importance of falls in maintaining a healthy life in older adults, accurate measurement of the frequency of falls is challenging because falls are an individual exper- ience in everyday life. Falls can only be self-reported and not objectively observed, rendering accurate measurement impossible. There is no validated or standard period for recall of falls, and therefore, no consensus on the optimal recall period. For this reason, different researchers have used different methods and periods ranging from weeks to years for recall ${ }^{14-18)}$. This situation arises from the fact that the validity of the self-reported incidence of falls cannot be measured. The reliability, however, can be indirectly measured through repetition of the same questions over different lengths of recall periods.

We hypothesized that quarterly surveys over a year would be superior to a 1-time annual survey for older adults to recall the number of falls they experienced more accurately; therefore, we aimed to compare the characteristics and outcomes of older adults who experienced falls as recalled in the past 1 year versus every 3 months over a year to suggest a more optimal recall period for large cohort studies in the future. 


\section{METHODS}

\section{Study Population}

We recruited community-dwelling older adults aged 65 years and older at a municipal senior welfare center in Seoul, Korea between January 18, 2016 and January 29, 2016. Older adults who voluntarily provided informed consent and scored 24 points or higher on the Korean version of Mini-Mental State Examination (K-MMSE) after adjusting for educational level were deemed eligible for participation. The participants were expected to undergo a fall risk assessment during baseline evaluation and respond to telephone surveys regarding their recent history of falls over the following year. Therefore, older adults who reported having serious orthopedic problems, disabling lumbar pain, inability to stand alone, serious visual disorder, uncontrolled hypertension (defined as systolic blood pressure more than $140 \mathrm{mmHg}$ or diastolic blood pressure more than $90 \mathrm{mmHg}$ ) or hypotension (defined as systolic blood pressure less than $90 \mathrm{mmHg}$ ), uncontrolled hyperglycemia (defined as a preprandial blood glucose level more than $140 \mathrm{mg} / \mathrm{dL}$ ) or hypoglycemia (defined as a postprandial blood glucose level less than $70 \mathrm{mg} / \mathrm{dL}$ ), a history of ischemic or hemorrhagic stroke, syncope, myocardial infarction, heart failure, atrial fibrillation, liver failure, renal failure, dementia, insulin injection or surgery within the last 3 months, or psychiatric diseases (e.g., schizophrenia, epilepsy, alcohol use disorder, drug abuse, and eating disorders) were excluded. Participants who reported a fall in either survey method were regarded as fallers, whereas participants who did not report any fall in both survey methods were regarded as nonfallers.

\section{Baseline Evaluation}

At baseline, the participants were asked about their sociodemographic information, level of weekly exercise in terms of the Korean version of the International Physical Activity Questionnaire, detailed medical history, including medication use, and history of a fall and any associated injury within the past year. In addition, a thorough history review and physical examination was performed by trained physicians, and the Berg balance evaluation, short physical performance battery (SPPB), and timed up and go test for fall risk assessment was performed in all participants.

\section{Survey Elements}

A fall was defined as involuntary landing of a body part, other than a foot, from one level to a lower level, such as the floor or the ground. Involuntary kneeling, landing a hand on a table during sudden tilting, landing on a hand after a slip, and losing balance and landing on the hip were given as examples of a fall. All eligible participants were surveyed via the telephone every 3 months over the following year by trained interviewers. During each survey, the participants were asked whether they experienced any fall over the 3 months after the last survey, and if they fell, how many times they fell, why they fell, whether they experienced any consequent injury, and about the site of fracture in the case of a fracture. During the first quarterly survey, the participants were also asked whether they had any fear of falling during everyday life, and whether fear of falling prevented them from going out. The participants were asked to recall the total number of falls over the last 1 year during the fourth quarterly survey. If the participant reported falling without recalling the exact number, the number was set as the rounded up number of the average number of falls of other fallers during the same period. If the participant recalled the number of falls as a range and not an exact number, the larger number was chosen as the number of falls.

\section{Statistical Analysis}

The primary outcome of this study was the report of any fall within 1 year. The baseline characteristics of the fallers and nonfallers were first identified. Recall discrepancy between participants with identical recall and discrepant recall was compared in relation to the number of falls by the sum of all quarterly recalls, and by 1 -year recall. Then, we further analyzed the fall-related characteristics of fallers who fell only once and fallers who fell more than once to verify their differences. We assessed the differences between continuous variables using analysis of variance and the differences between categorical variables using the chi-square test or Fisher exact test.

All statistical analyses were conducted using $R$, version 3.4.0 (R Foundation for Statistical Computing, Vienna, Austria).

\section{Ethics Statement}

This study was approved by the Institutional Review Board (approval number: KHUH 2017-09-045) of Kyung Hee University Hospital.

\section{RESULTS}

Initially, 317 eligible participants were enrolled in the study, and 247 of them completed all 4 quarterly follow-ups and the final 1-year follow-up. Fifty-eight participants reported at least 1 fall during the follow-up period in either method of surveys. The numbers of falls recalled in 4 quarterly surveys and in the 1-year survey were the same in 29 participants and discrepant in the other 29 participants. Fig. 1 illustrates the detailed numerical flow of participants. 
There was no statistically significant difference in age and sex between the fallers and nonfallers, with the mean age of the participants being 76.85 years and women constituting for $68.8 \%$ of them (Table 1). Other baseline characteristics also were not significantly different, except for the type of residence, history of fall in the previous year, Berg balance score (BBS), and presence of a sleep disorder. Approximately $31.2 \%$ of nonfallers and $20.7 \%$ of fallers resided in apartment buildings ( $p=0.049)$, and fallers had a higher rate of falls in the previous year $(32.8 \%)$ than nonfallers $(14.3 \%)(p=0.003)$. The BBS (mean \pm standard deviation) was lower in fallers $(51.10 \pm 5.02)$ than in nonfallers $(52.92 \pm 3.08)(p=0001)$. While other comorbidities did not show a significant difference, the rate of sleep disorder was higher in fallers (20.7\%) than in nonfaller $(9.5 \%)(p=0.041)$.

Among 58 fallers, 56 participants reported 80 falls in quarterly surveys, and 47 participants reported 67 falls in the 1-year survey, indicating an approximately 19\% reduction in the rate of recall in the 1-year survey (Table 2). Eleven participants reported no falls in the 1-year survey despite reporting 1 or more falls in quarterly surveys. Notably, 9 of them reported falling only during the first quarterly survey. Among fallers who did not fall again after each quarter, 10 of 16 fallers (62.5\%) who did not report a fall after the first quarter showed a higher recall discrepancy than 4 of $8(50.0 \%)$,

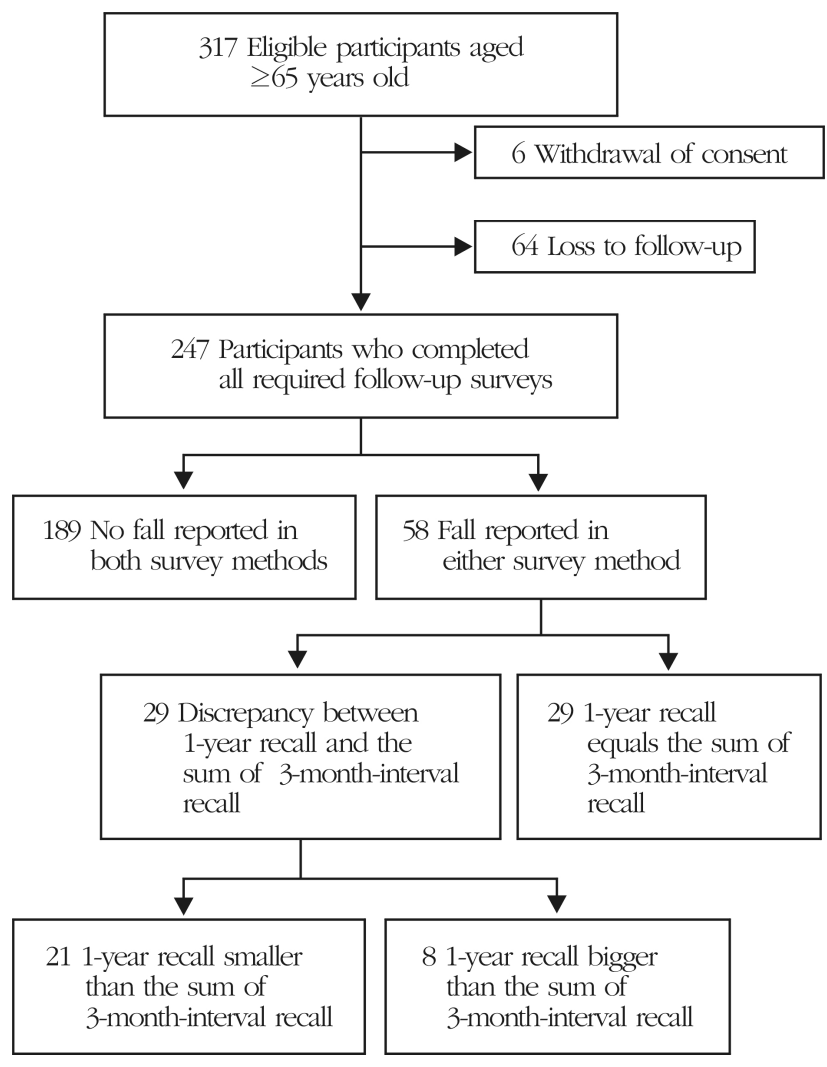

Fig. 1. Flow chart of eligible participants.
4 of $13(30.8 \%)$, and 9 of 19 fallers (47.4\%) during the second, third, and fourth quarter, respectively. Thirty-nine fallers reported falling only during 1 quarterly survey, while 15 fallers reported falling during 2 quarterly surveys, 1 faller reported falling during 3 quarterly surveys, and 1 faller reported falling during all 4 quarterly surveys. Among 56 fallers according to the sum of quarterly surveys, 35 fell once and 21 fell more than once. Among participants who fell more than once, 16 of $21(76.2 \%)$ showed recall discrepancy against the 1-year recall, while only 11 of 351 -time fallers $(31.4 \%)$ showed recall discrepancy.

Participants with identical or discrepant recall between the sum of quarterly recalls and 1-year recall revealed that participants who fell more than once had higher recall discrepancy (16 of 21 participants [76.2\%]) than those who fell only once (11 of 35 participants [31.4\%]) according to the sum of quarterly surveys (Table 3 ). Most participants (24 [82.8\%]) with identical recall reported 1 fall only.

The participants who fell only once and participants who fell more than once per year according to the sum of quarterly surveys were further analyzed to identify the factors that may contribute to recall discrepancy (Table 4). The results did not show a statistically significant difference in most aspects of fall-related characteristics, except that the participants who fell more than once had more experience of falling within 1 year before the study $(p=0.026)$, and more participants who fell only once were on medication $(p=0.019)$.

\section{DISCUSSION}

When asked about the past 1 year, only half of older adults gave the same number of falls as they recalled at the 3-month interval and at the 1-year interval, and 1 of 5 older adults did not remember falling at all after 3 to 9 months. Older adults who fell more than once per year had poorer recall of the number of falls than those who fell only once. The results of our study indicate significant memory decay when surveying fall-related events over a 1-year period and support a shorter period of 3 months and less for a more accurate measurement of falls.

\section{Clinical Implication of the Results}

There are many methods other than a telephone survey, such as a calendar ${ }^{14)}$, patient diary ${ }^{15)}$, postcard ${ }^{16)}$, and mailed questionnaire $^{177}$, that are used widely and often for prospective recording during study participation. Although it is generally accepted that immediate recording in a calendar, diary, and postcard yields more accurate and a higher rate of report than retrospective recall, these methods require much dedication and prior education of the participants. Therefore, telephone surveys remain the best practical option for retro- 
spective report, especially in studies of a large sample size.

Notably, 9 of 25 participants who reported falling during the first quarterly survey did not recall any fall in the final 1-year survey in our study. It is reasonable to assume that these participants had forgotten the event of a fall 9 or more months ago, and the sum of quarterly recalls provides a more accurate report than 1-year recall. This assumption is again stressed by the finding that the participants who only fell during the first 3 months showed higher recall discrepancy of $62.5 \%$ than the rest, ranging from $30.8 \%$ to

Table 1. Baseline characteristics of participants with and without a history of falls

\begin{tabular}{|c|c|c|c|c|}
\hline \multirow{2}{*}{ Characteristic } & \multicolumn{3}{|c|}{ History of falls } & \multirow{2}{*}{ p-value* } \\
\hline & Total $(n=247)$ & Yes $(n=58)$ & No $(n=189)$ & \\
\hline Age (yr) & $76.85 \pm 4.89$ & $77.53 \pm 5.06$ & $76.63 \pm 4.83$ & 0.221 \\
\hline Sex & & & & 0.851 \\
\hline Male & $77(31.2)$ & $17(29.3)$ & $60(31.7)$ & \\
\hline Female & $170(68.8)$ & $41(70.7)$ & $129(68.3)$ & \\
\hline Smoker & $20(8.1)$ & $2(3.4)$ & $18(9.5)$ & 0.227 \\
\hline Alcohol consumers & $61(24.7)$ & $10(17.2)$ & $51(27.0)$ & 0.183 \\
\hline$\geq$ Moderate level of exercise & $87(35.2)$ & $18(31.0)$ & $69(36.5)$ & 0.544 \\
\hline Use of an assistive device & $12(4.9)$ & $5(8.6)$ & $7(3.7)$ & 0.240 \\
\hline BMI $\left(\mathrm{kg} / \mathrm{m}^{2}\right)$ & & & & 0.716 \\
\hline$<18.5$ & $7(2.8)$ & $1(1.7)$ & $6(3.2)$ & \\
\hline $18.5-25$ & $110(44.5)$ & $28(48.3)$ & $82(43.4)$ & \\
\hline$\geq 25$ & $130(52.6)$ & $29(50.0)$ & $101(53.4)$ & \\
\hline K-MMSE score & & & & 0.210 \\
\hline$\geq 27$ & $139(56.3)$ & $28(48.3)$ & $111(58.7)$ & \\
\hline $24-26$ & $108(43.7)$ & $30(51.7)$ & $76(40.2)$ & \\
\hline Elementary school graduate & $199(80.6)$ & $45(77.6)$ & $154(81.5)$ & 0.641 \\
\hline Living alone & $149(60.3)$ & $17(29.3)$ & $81(42.9)$ & 0.091 \\
\hline Type of residence & & & & 0.049 \\
\hline Apartment & $71(28.7)$ & $12(20.7)$ & $59(31.2)$ & \\
\hline Multihousing & $70(28.3)$ & $18(31.0)$ & $52(27.5)$ & \\
\hline Single housing & $102(41.3)$ & $25(43.1)$ & $77(40.7)$ & \\
\hline Other & $4(1.6)$ & $3(5.2)$ & $1(0.5)$ & \\
\hline Falls in the previous year & $46 \pm 18.6$ & $19 \pm 32.8$ & $27 \pm 14.3$ & 0.003 \\
\hline Berg balance score & $52.49 \pm 3.70$ & $51.10 \pm 5.02$ & $52.92 \pm 3.08$ & 0.001 \\
\hline SPPB & $9.32 \pm 1.98$ & $8.88 \pm 2.26$ & $9.46 \pm 1.88$ & 0.051 \\
\hline Timed up and go test (sec) & & & & 0.450 \\
\hline$<13.5$ & $206(83.4)$ & $46(79.3)$ & $160(84.7)$ & \\
\hline$\geq 13.5$ & $41(16.6)$ & $12(20.7)$ & $29(15.3)$ & \\
\hline \multicolumn{5}{|l|}{ Current medication use } \\
\hline All types of medication & $197(79.8)$ & $49(84.5)$ & $148(78.3)$ & 0.403 \\
\hline Sedative & $7(2.8)$ & $2(3.4)$ & $5(2.6)$ & 1.000 \\
\hline Hypnotic & $17(6.9)$ & $6(10.3)$ & $11(5.8)$ & 0.371 \\
\hline Antidepressant & $15(6.1)$ & $6(10.3)$ & $9(4.8)$ & 0.214 \\
\hline \multicolumn{5}{|l|}{ Comorbidities } \\
\hline Hearing difficulty & $13(5.3)$ & $2(3.4)$ & $11(5.8)$ & 0.710 \\
\hline Depression & $14(5.7)$ & $6(10.3)$ & $8(4.3)$ & 0.154 \\
\hline Sleep disorder & $30(12.1)$ & $12(20.7)$ & $18(9.5)$ & 0.041 \\
\hline
\end{tabular}

Values presented as a mean \pm standard deviation or number (\%).

BMI, body mass index; K-MMSE, Korean Mini-Mental State Examination; SPPB, short physical performance battery.

"p-values were obtained by analysis of variance for continuous variables and the chi-square test or Fisher exact test for categorical variables. 
$50.0 \%$. These results are supported by previous studies that compared self-recorded calendars and telephone surveys at the end of the study. Hannan et al. ${ }^{19)}$ compared quarterly telephone recalls with monthly fall calendars and concluded that quarterly telephone recall underestimates the number of fallers by as much as $25 \%$ compared to keeping a self-reported calendar. Additionally, Mackenzie et al. ${ }^{20)}$ compared 6-month recall for falls and a prospective calendar-reported method and concluded that 33 of 76 participants (43.4\%) who reported falling on calendars did not recall falling during the final survey at 6 months. These findings indicate the superiority of recording calendars over retrospective recall, but they also highlight the extremely high rate of recall discrepancy in older adults within a few months.

The results of our study that 3-month recall is much more accurate than 1-year recall may not be surprising. However, a staggering number of regional and national studies on older adults aged 65 years and older rely on the participants' 1-year recall for fall-related events ${ }^{1,21,22)}$. The 2014 Behavioral Risk Factor Surveillance System survey in the United States reported that $28.7 \%$ of older adults experienced falls within a year, and $37.5 \%$ of them required medical treatment or experienced restriction of activities for at least 1 day $^{1)}$; the 2009 New South Wales Falls Prevention Baseline Survey in Australia reported that $25.6 \%$ of older adults experienced falls within a year, and $20 \%$ of them visited a hospital for treatment ${ }^{21)}$; and the 2014 Survey of Living Conditions and Welfare Needs of Korean Older Persons in Korea reported that $25.1 \%$ of older adults experienced falls within a year, and $63.4 \%$ of them sought medical attention ${ }^{22)}$. Frequent use of the 1-year recall is explained by the investigators' intention to present the annual incidence of falls with limited resources and time. However, such surveys may underestimate the number of older adults who experience falls annually by $19 \%$ and more according to the results of our study.

The participants who fell more than once had a much higher recall discrepancy (76.2\%) than those who only fell once (31.4\%). The participants who fell more than once recalled ever falling just as well as participants who fell only once. The only difference was that the former had much trouble remembering how many times they fell. This is understandable considering the difficulty of recalling the number of events within a year, especially if they did not result in devastating consequences, which is often the case with falls. Therefore, the data regarding the number of falls within a year may be subject to questions of accuracy in surveys using 1 -year recall. Further analysis of the participants who fell more than once and showed a higher recall discrepancy re-

Table 2. Summary of quarterly and 1-year survey results

\begin{tabular}{|c|c|c|c|c|c|c|}
\hline & 1st quarter & 2nd quarter & 3rd quarter & 4th quarter & Sum of 4 quarters & 1-Year recall \\
\hline No. of falls & 31 & 16 & 19 & 21 & 80 & 67 \\
\hline No. of fallers & 25 & 13 & 19 & 19 & 56 & 47 \\
\hline New fallers in the quarter & 25 & 7 & 13 & 11 & - & - \\
\hline $\begin{array}{l}\text { Fallers without a subsequent fall after the } \\
\text { corresponding quarter }\end{array}$ & 16 & 8 & 13 & 19 & - & - \\
\hline Ratio of fallers with recall discrepancy* & $10 / 16(62.5)$ & $4 / 8(50.0)$ & $4 / 13(30.8)$ & $9 / 19(47.4)$ & - & - \\
\hline
\end{tabular}

Values are presented as a number or number (\%).

*The ratio of fallers with recall discrepancy was computed only among fallers without a subsequent fall after the corresponding quarter using the number of fallers with recall discrepancy as the numerator.

Table 3. Recall discrepancy between participants with identical recall and discrepant recall as presented by the number of falls by the sum of all quarterly recalls and by 1 -year recall

\begin{tabular}{|c|c|c|c|c|}
\hline & \multicolumn{3}{|c|}{ Recall discrepancy } & \multirow{2}{*}{ p-value* } \\
\hline & Total $(n=58)$ & Identical $(n=29)$ & Discrepant $(n=29)$ & \\
\hline No. of falls by the sum of all quarterly recalls & & & & 0.002 \\
\hline 0 & $2(3.4)$ & $0(0)$ & $2(6.9)$ & \\
\hline 1 & $35(60.3)$ & $24(82.8)$ & $11(37.9)$ & \\
\hline$\geq 2$ & $21(36.2)$ & $5(17.2)$ & $16(55.2)$ & \\
\hline No. of falls by 1 -year recall & & & & $<0.001$ \\
\hline 0 & $11(19.0)$ & $0(0)$ & $11(37.9)$ & \\
\hline 1 & $33(56.9)$ & $24(82.8)$ & $9(31.0)$ & \\
\hline$\geq 2$ & $14(24.1)$ & $5(17.2)$ & $9(31.0)$ & \\
\hline
\end{tabular}

Values are presented as a number (\%).

"p-values were obtained by the Fisher exact test. 
vealed that they also experienced more falls within 1 year before the study, which suggests that they had a greater risk of falling than those who only fell once. However, the factors associated with their increased recall discrepancy could not be pursued further due to the limitation of a small number of participants.

One interesting finding was that older adults living in apart- ment buildings experienced significantly fewer falls than those living in other types of housing $(p=0.049)$. One regional study in Korea reported that most falls occurred while walking on flat ground or using stairs ${ }^{13}$. Considering that the residents of apartment buildings are prone to using elevators whereas other types of housing involve more frequent use of stairs, the use of stairs might have contributed to the difference.

Table 4. Comparison of participants who fell only once and participants who fell more than once per a year according to the sum of quarterly recalls

\begin{tabular}{|c|c|c|c|c|}
\hline \multirow{2}{*}{ Variable } & \multicolumn{3}{|c|}{ No. of falls } & \multirow{2}{*}{ p-value* } \\
\hline & Total $(n=56)$ & $1(n=35)$ & $\geq 2(n=21)$ & \\
\hline Age(yr) & $77.46 \pm 5.14$ & $78.03 \pm 4.29$ & $76.52 \pm 6.31$ & 0.293 \\
\hline Sex & & & & 0.359 \\
\hline Male & $16(28.6)$ & $8(22.9)$ & $8(38.1)$ & \\
\hline Female & $40(71.4)$ & $27(77.1)$ & $13(61.9)$ & \\
\hline Smoker & $2(3.6)$ & $1(2.9)$ & $1(4.8)$ & 1.000 \\
\hline Alcohol consumer & $9(16.1)$ & $7(20.0)$ & $2(9.5)$ & 0.511 \\
\hline K-MMSE score & & & & 1.000 \\
\hline$\geq 27$ & $28(50.0)$ & $17(48.6)$ & $11(52.4)$ & \\
\hline $24-26$ & $28(50.0)$ & $18(51.4)$ & $10(47.6)$ & \\
\hline Elementary school graduate & $43(76.8)$ & $26(74.3)$ & $17(81.0)$ & 0.806 \\
\hline Living alone & $17(30.4)$ & $10(28.6)$ & $7(33.3)$ & 0.940 \\
\hline Type of residence & & & & 0.450 \\
\hline Apartment & $11(19.6)$ & $6(17.1)$ & $5(23.8)$ & \\
\hline Multihousing & $19(33.9)$ & $10(28.6)$ & $8(38.1)$ & \\
\hline Single housing & $24(42.9)$ & $16(45.7)$ & $8(38.1)$ & \\
\hline Other & $3(5.4)$ & $3(8.6)$ & $0(0.0)$ & \\
\hline Fall in the last year & $1.13 \pm 0.84$ & $0.94 \pm 0.64$ & $1.45 \pm 1.04$ & 0.026 \\
\hline Berg balance score & $51.14 \pm 5.09$ & $51.43 \pm 4.42$ & $50.67 \pm 6.13$ & 0.592 \\
\hline SPPB & $8.93 \pm 2.24$ & $9.17 \pm 2.09$ & $8.52 \pm 2.46$ & 0.299 \\
\hline Timed Up and Go test (sec) & & & & 0.339 \\
\hline$<13.5$ & $45(80.4)$ & $30(85.7)$ & $15(71.4)$ & \\
\hline$\geq 13.5$ & $11(19.6)$ & $5(14.3)$ & $6(28.6)$ & \\
\hline Injury & $35(62.5)$ & $23(65.7)$ & $12(57.1)$ & 0.722 \\
\hline Fear of falling in the first 3-month survey & & & & 0.601 \\
\hline None & $23(41.1)$ & $16(45.7)$ & $7(33.3)$ & \\
\hline Little & $17(30.4)$ & $11(31.4)$ & $6(28.6)$ & \\
\hline Substantially & $9(16.1)$ & $5(14.3)$ & $4(19.0)$ & \\
\hline Seriously & $7(12.5)$ & $3(8.6)$ & $4(19.0)$ & \\
\hline \multicolumn{5}{|l|}{ Current medication use } \\
\hline All types of medication & 47 (83.9) & $33(94.3)$ & $14(66.7)$ & 0.019 \\
\hline Sedative & $2(3.6)$ & $2(5.7)$ & $0(0)$ & 0.710 \\
\hline Hypnotic & $6(10.7)$ & $4(11.4)$ & $2(9.5)$ & 1.000 \\
\hline Antidepressant & $6(10.7)$ & $3(8.6)$ & $3(14.3)$ & 0.823 \\
\hline No. of comorbidities & $2.54 \pm 1.53$ & $2.54 \pm 1.42$ & $2.52 \pm 1.72$ & 0.964 \\
\hline
\end{tabular}

Values are presented as a mean \pm standard deviation or number (\%).

K-MMSE, Korean Mini-Mental State Examination; SPPB, short physical performance battery.

"p-values were obtained by analysis of variance for continuous variables and the chi-square test or Fisher exact test for categorical variables. 


\section{Strengths and Limitations of the Study}

We excluded patients with K-MMSE score less than 24 or a history of dementia and other psychiatric diseases that may significantly decrease the participants' ability to recall accurately, thereby targeting participants with normal cognitive functions. We also tried to identify any determining factor that contributes to recall discrepancy by comparing the 2 groups with identical recall and discrepant recall, although with not much significant findings other than the difference in the number of falls.

The foremost limitation is the lack of validity. We could not ascertain whether the participants really experienced falls, so we had to rely on their responses. However, this limitation is true of all relevant studies regardless of study design. We endeavored to minimize false reports of falls by enquiring about the relevant circumstances of falls. Furthermore, this study shares the common limitations of self-reported surveys, such as memory decay and recall bias. Especially, falls often end as a 1-time trivial event in many people, in which case the person may find it difficult to recall falling. In contrast, older adults who experience more serious injuries would recall better, increasing the relative rate of injuries. We collected the information regarding a fall only once for each given period, and not twice or more to verify the reproducibility of the responses. We considered that the participants may experience another fall after each survey, causing discrepancy in report due to additional falls reported in between the survey periods, not due to a lack of reproducibility. Another important limitation is selection bias. The participants were recruited at one senior center in downtown Seoul who could relatively ambulate freely on their own and participate in group activities. Additionally, only those who could complete the Berg balance evaluation and SPPB were included in the study. Therefore, the results of the study cannot represent the whole population of older people in Korea. Further, the number of participants was relatively small, and further in-depth analysis of the participants with recall discrepancy was limited. Another limitation to note is the loss to follow-up. About 20\% of the participants could not be reached via telephone by the end of the 1-year study. Finally, there may be some overlap of memory in participants who reported falls during 2 or more consecutive quarterly surveys. The investigators were trained to overcome these limitations as much as possible.

In conclusion, repeated surveys with a shorter recall period of 3 months and less may yield a more accurate measurement of falls than a survey with a recall period of 1 year. If 1-year recall must be used to obtain the annual incidence of falls, the investigator must be aware that it may underestimate the incidence of falls by $19 \%$ or more, and older adults often find it difficult to remember how many times they fell within a 1-year period in the case of more than one fall. Further studies with a larger sample size and varying recall periods are required to standardize the method of measuring falls.

Conflicts of Interest Disclosures: The researchers claim no conflicts of interest.

\section{Acknowledgments}

This study was funded by InBody Co., Ltd.

\section{REFERENCES}

1. Bergen G, Stevens MR, Burns ER. Falls and fall injuries among adults aged $\geq 65$ years - United States, 2014. MMWR Morb Mortal Wkly Rep 2016;65:993-8.

2. Blake AJ, Morgan K, Bendall MJ, Dallosso H, Ebrahim SB, Arie $\mathrm{TH}$, et al. Falls by elderly people at home: prevalence and associated factors. Age Ageing 1988;17:365-72.

3. Hill K, Vrantsidis F, Haralambous B, Fearn M, Smith R, Murray $\mathrm{K}$, et al. An analysis of research on preventing falls and falls injury in older people: community, residential care and hospital settings (2004 update). Report to the Australian Government, Department of Health and Ageing, Injury Prevention Section by the National Ageing Research Institute. Canberra (Australia): Commonwealth of Australia; 2004.

4. Scuffham P, Chaplin S, Legood R. Incidence and costs of unintentional falls in older people in the United Kingdom. J Epidemiol Community Health 2003;57:740-4.

5. Rubenstein LZ. Falls in older people: epidemiology, risk factors and strategies for prevention. Age Ageing 2006;35 Suppl 2:ii3741.

6. Stevens JA, Corso PS, Finkelstein EA, Miller TR. The costs of fatal and non-fatal falls among older adults. Inj Prev 2006;12: 290-5.

7. Abrahamsen B, van Staa T, Ariely R, Olson M, Cooper C. Excess mortality following hip fracture: a systematic epidemiological review. Osteoporos Int 2009;20:1633-50.

8. Koval KJ, Skovron ML, Aharonoff GB, Meadows SE, Zuckerman JD. Ambulatory ability after hip fracture. A prospective study in geriatric patients. Clin Orthop Relat Res 1995;(310): 150-9.

9. Kitamura S, Hasegawa Y, Suzuki S, Sasaki R, Iwata H, Wingstrand $\mathrm{H}$, et al. Functional outcome after hip fracture in Japan. Clin Orthop Relat Res 1998;(348):29-36.

10. van Balen R, Steyerberg EW, Polder JJ, Ribbers TL, Habbema JD, Cools HJ. Hip fracture in elderly patients: outcomes for function, quality of life, and type of residence. Clin Orthop Relat Res 2001;(390):232-43.

11. Lee JH, Ahn EM, Kim GE, Jeong YK, Kim JH, Kim JH, et al. The effects of falling and fear of falling on daily activity levels in the elderly residing in Korean rural community. J Korean Geriatr Soc 2009;13:79-88.

12. Vellas BJ, Wayne SJ, Romero LJ, Baumgartner RN, Garry PJ. Fear of falling and restriction of mobility in elderly fallers. Age Ageing 1997;26:189-93. 
13. Lim JY, Park WB, Oh MK, Kang EK, Paik NJ. Falls in a proportional region population in Korean elderly: incidence, consequences, and risk factors. J Korean Geriatr Soc 2010;14:8-17.

14. Tromp AM, Pluijm SM, Smit JH, Deeg DJ, Bouter LM, Lips P. Fall-risk screening test: a prospective study on predictors for falls in community-dwelling elderly. J Clin Epidemiol 2001;54: 837-44.

15. Hill AM, Hoffmann T, Haines TP. Circumstances of falls and falls-related injuries in a cohort of older patients following hospital discharge. Clin Interv Aging 2013;8:765-74.

16. Dawson-Hughes B, Harris SS, Krall EA, Dallal GE. Effect of calcium and vitamin D supplementation on bone density in men and women 65 years of age or older. N Engl J Med 1997;337: 670-6.

17. Wagner EH, LaCroix AZ, Grothaus L, Leveille SG, Hecht JA, Artz K, et al. Preventing disability and falls in older adults: a population-based randomized trial. Am J Public Health 1994; 84:1800-6.

18. Hauer K, Lamb SE, Jorstad EC, Todd C, Becker C; PROFANE-
Group. Systematic review of definitions and methods of measuring falls in randomised controlled fall prevention trials. Age Ageing 2006;35:5-10.

19. Hannan MT, Gagnon MM, Aneja J, Jones RN, Cupples LA, Lipsitz LA, et al. Optimizing the tracking of falls in studies of older participants: comparison of quarterly telephone recall with monthly falls calendars in the MOBILIZE Boston Study. Am J Epidemiol 2010;171:1031-6.

20. Mackenzie L, Byles J, D'Este C. Validation of self-reported fall events in intervention studies. Clin Rehabil 2006;20:331-9.

21. Milat AJ, Watson WL, Monger C, Barr M, Giffin M, Reid M. Prevalence, circumstances and consequences of falls among community-dwelling older people: results of the 2009 NSW Falls Prevention Baseline Survey. N S W Public Health Bull 2011;22: 43-8.

22. Jeong G, Oh Y, Kang E, Kim J, Sunwoo D, Oh M, et al. 2014 Survey of living conditions and welfare needs of Korean Older persons. Sejong (Korea): Korea Institute for Health and Social Affairs; 2014. 